\title{
Some new periodic Golay pairs
}

\author{
Dragomir Ž. Đoković 1, Ilias S. Kotsireas 2
}

\begin{abstract}
Periodic Golay pairs are a generalization of ordinary Golay pairs. They can be used to construct Hadamard matrices. A positive integer $v$ is a (periodic) Golay number if there exists a (periodic) Golay pair of length $v$. Taking into the account the results obtained in this note and yet unpublished new result [9], there are only seven known periodic Golay numbers which are definitely not Golay numbers, namely 34, 50, 58, 68, 72, 74, 82 . We construct here periodic Golay pairs of lengths $74,122,164,202,226$. It is apparently unknown whether 122,164,202,226 are Golay numbers. The smallest length for which the existence of periodic Golay pairs is undecided is now 90.
\end{abstract}

\section{Preliminaries}

Let $v$ be a positive integer and $\mathbf{Z}_{v}=\{0,1, \ldots, v-1\}$ the ring of integers modulo $v$. Let $A=a_{0}, a_{1}, \ldots, a_{v-1}$ be a binary, i.e., $\{ \pm 1\}$-sequence of length $v$. The periodic autocorrelation function $\mathrm{PAF}_{A}: \mathbf{Z}_{v} \rightarrow \mathbf{Z}$ and the nonperiodic autocorrelation function $\mathrm{NAF}_{A}: \mathbf{Z} \rightarrow \mathbf{Z}$ of $A$ are defined by

$$
\begin{aligned}
\operatorname{PAF}_{A}(s) & =\sum_{i=0}^{v-1} a_{i} a_{i+s} \quad(\bmod v) \\
\operatorname{NAF}_{A}(s) & =\sum_{i \in \mathbf{Z}} a_{i} a_{i+s}
\end{aligned}
$$

(In the nonperiodic case we set $a_{i}=0$ when $i<0$ or $i \geq v$.) Note that

$$
\operatorname{PAF}_{A}(s)=\mathrm{NAF}_{A}(s)+\mathrm{NAF}_{A}(v-s), \quad s \in \mathbf{Z}_{v} .
$$

A Golay pair is an ordered pair $(A, B)$ of binary sequences of length $v$ such that $\operatorname{NAF}_{A}(s)+$ $\operatorname{NAF}_{B}(s)=0$ for $s \neq 0$. Similarly, a periodic Golay pair is an ordered pair $(A, B)$ of binary sequences of length $v$ such that $\operatorname{PAF}_{A}(s)+\operatorname{PAF}_{B}(s)=0$ for $s \neq 0$. It follows from (1) that any Golay pair is also a periodic Golay pair. Periodic Golay pairs are also known as complementary binary sequences. They can be used to construct Hadamard matrices of order $2 v$ (see subsection 1.1 below). Thus, if $v>1$ is a periodic Golay number then $v$ must be even.

Periodic Golay pairs can be viewed as a particular case of supplementary difference sets (SDS). Let us recall some facts which will be used in our constructions, see e.g. [6, Proposition $1]$.

\footnotetext{
${ }^{1}$ University of Waterloo, Department of Pure Mathematics and Institute for Quantum Computing, Waterloo, Ontario, N2L 3G1, Canada, e-mail: djokovic@uwaterloo.ca

${ }^{2}$ Wilfrid Laurier University, Department of Physics \& Computer Science, Waterloo, Ontario, N2L 3C5, Canada, e-mail: ikotsire@wlu.ca
} 
First we have to recall the definition of SDS. Let $k_{1}, \ldots, k_{t}$ be positive integers and $\lambda$ an integer such that $\lambda(v-1)=\sum_{i=1}^{t} k_{i}\left(k_{i}-1\right)$, and let $X_{i}$ be a subset of $\mathbf{Z}_{v}$ with cardinality $\left|X_{i}\right|=k_{i}, i \in\{1,2, \ldots, t\}$.

Definition 1 We say that $\left(X_{1}, \ldots, X_{t}\right)$ are supplementary difference sets with parameters $\left(v ; k_{1}, \ldots, k_{t} ; \lambda\right)$, if for every nonzero element $c \in \mathbf{Z}_{v}$ there are exactly $\lambda$ ordered pairs $(a, b)$ such that $a-b=c(\bmod v)$ and $\{a, b\} \subseteq X_{i}$ for some $i \in\{1,2, \ldots, t\}$.

For convenience, we shall refer to the sets $X_{1}, \ldots, X_{t}$ as the base blocks of this SDS. To any SDS with parameters $\left(v ; k_{1}, \ldots, k_{t} ; \lambda\right)$ we attach an additional parameter $n$ defined by $n=k_{1}+\cdots+k_{t}-\lambda$. (SDSs with $t=1$ are known as cyclic difference sets.)

There is a bijection from the set of all binary sequences of length $v$ to the set of all subsets of $\mathbf{Z}_{v}$ which assigns to the sequence $A$ the subset $\left\{i \in \mathbf{Z}_{v}: a_{i}=-1\right\}$. If $(A, B)$ is a periodic Golay pair, then the corresponding pair of subsets $(X, Y)$ of $\mathbf{Z}_{v}$ is an SDS whose parameters $(v ; r, s ; \lambda)$ satisfy the equation $v=2 n$. (Recall that $n=r+s-\lambda$ in this case.) The converse is also true. Moreover, if $a=v-2 r$ and $b=v-2 s$ then $a^{2}+b^{2}=2 v$. In particular, $v$ must be a sum of two squares.

\subsection{Periodic Golay pairs and Hadamard matrices}

One of the reasons that periodic Golay pairs are useful is that they can be used to construct Hadamard matrices. Let us detail the relevant construction. Suppose that two binary sequences $A, B$ of length $v$ that form a periodic Golay pair are given. Then one can construct the associated $v \times v$ circulant matrices $C_{A}, C_{B}$ whose first rows are the sequences $A, B$ respectively. These two circulant matrices satisfy the matrix equation

$$
C_{A} C_{A}^{t}+C_{B} C_{B}^{t}=(2 v) I_{v}
$$

where $t$ denotes transposition and $I_{v}$ denotes the $v \times v$ unit matrix. Using these two circulant matrices, a Hadamard matrix of order $2 v$ can be constructed as

$$
H_{2 v}=\left[\begin{array}{c|c}
C_{A} & C_{B} \\
\hline-C_{B}^{t} & C_{A}^{t}
\end{array}\right] .
$$

We refer the reader to [14] for more details and additional Hadamard matrix constructions.

\section{Known Golay and periodic Golay numbers}

If $\alpha, \beta, \gamma$ are nonnegative integers, then it is well known that $2^{\alpha} 10^{\beta} 26^{\gamma}$ is a Golay number. No additional Golay numbers have been found so far. Moreover, there are no other Golay numbers in the range $1,2, \ldots, 100$ (see [2]).

We have already mentioned in Section 1 that the periodic Golay number $v>1$ must be even and a sum of two squares. The following two important necessary conditions for Golay and periodic Golay numbers have been proved more than twenty years ago. 
Theorem 1 (Eliahou-Kervaire-Saffari [12]) A Golay number is not divisible by any prime $p \equiv 3$ $(\bmod 4)$.

Theorem 2 (Arasu-Xiang [1, Corollary 3.6]) If $v=p^{t} u>1$ is a periodic Golay number, $p \equiv 3$ (mod 4$)$ is a prime number, and $(p, u)=1$ then $u \geq 2 p^{t / 2}$.

(Since $v$ is a sum of two squares, the exponent $t$ is an even integer.)

By applying this theorem to the integers in the range $1,2, \ldots, 500$, we deduce that the numbers $18,36,98,162,242,324,392,484,490$ are not periodic Golay although each of them is even and a sum of two squares.

It has been shown very recently [10, Theorem 1] that the product of a Golay number and a periodic Golay number is again a periodic Golay number. More precisely, the authors of that paper show how to "multiply" an ordinary Golay pair of length $g$ and a periodic Golay pair of length $d$ to obtain a periodic Golay pair of length $g d$. Consequently, there are infinitely many periodic Golay numbers which are not of the form $2^{\alpha} 10^{\beta} 26^{\gamma}$. We point out that this "multiplication theorem" is an easy consequence of [11, Theorems 13 and 14].

As noted in the abstract, there are currently only seven periodic Golay numbers for which we know that they are not Golay numbers. More specifically we have that:

- The first periodic Golay pair whose length, 34, is not a Golay number has been found in 1998 by Đoković [3]. (In fact two non-equivalent such pairs were found.)

- Periodic Golay pairs of length 50 have been found by Đoković [4] and Kotsireas and Koukouvinos [13].

- Periodic Golay pairs of length 58 have been found by Đoković and Kotsireas [6].

- Several periodic Golay pairs of length 68 have been constructed recently in [8]. Such pairs can be constructed also by using the method described in [10].

- Periodic Golay pairs of length 72 have been constructed recently [9].

- For examples of periodic Golay pairs of length 74 see the next section.

- Periodic Golay pair of length 82 has been found in 2008 by Vollrath [15] (see also the next section).

\section{New periodic Golay pairs}

We have constructed several new periodic Golay pairs, and we deduce that 74, 122, 164, 202, 226 are periodic Golay numbers. As $74<100$ we know that 74 is not a Golay number. It is apparently unknown whether 122,164,202, 226 are Golay numbers. We also construct periodic Golay pairs of length 82, not equivalent to the known one [15]. In all cases below, the nonequivalence is established by the method described in [4]. 
The SDSs $(X, Y)$ listed below are given by using the following compact notation. The parameter set is $(v ; r, s ; \lambda)$ with $n=r+s-\lambda$ and $v=2 n$. In each case, we make use of a nontrivial subgroup $H$ of the group of units $\mathbf{Z}_{v}^{\star}$ of the ring $\mathbf{Z}_{v}$. This subgroup acts on $\mathbf{Z}_{v}$ by multiplication modulo $v$. The orbit of $H$ containing $j \in \mathbf{Z}_{v}$ is given by $H \cdot j=\{h j$ $(\bmod v): h \in H\}$. The base blocks $X$ and $Y$ are composed of orbits of $H$. Thus we have

$$
X=\bigcup_{j \in J} H \cdot j, \quad Y=\bigcup_{k \in K} H \cdot k,
$$

where $J, K \subseteq \mathbf{Z}_{v}$. Instead of listing the elements of $X$ and $Y$ we shall list only their index sets $J$ and $K$, respectively. We note that, as a representative $j$ of an orbit $H \cdot j$ we always choose the smallest integer in that orbit.

A short description of the method we used to construct the required SDSs and some computational details are given in section 4 .

\subsection{Periodic Golay pairs of length 74}

The parameter set is $(74 ; 36,31 ; 30)$, with $n=37$. Using the subgroup $H=\{1,47,63\}$ of $Z_{74}^{\star}$, we give two non-equivalent solutions:

$$
\begin{aligned}
J & =\{1,4,6,7,9,12,22,23,28,29,34,42\} \\
K & =\{1,2,4,6,9,12,17,21,22,37,55\} \\
J & =\{1,2,3,6,7,21,22,23,28,29,34,55\} \\
K & =\{2,4,5,7,9,10,17,21,34,37,42\}
\end{aligned}
$$

\subsection{Periodic Golay pairs of length 82}

The parameter set is $(82 ; 45,36 ; 40)$, with $n=41$. Using the subgroup $H=\{1,37,51,57,59\}$ of $Z_{82}^{\star}$, we give two non-equivalent solutions:

$$
\begin{aligned}
J & =\{1,2,11,12,15,17,22,23,30\} \\
K & =\{1,4,10,12,17,22,23,41\} \\
J & =\{1,2,3,6,8,12,17,23,30\} \\
K & =\{3,5,6,12,17,22,30,41\}
\end{aligned}
$$




\subsection{Periodic Golay pairs of length 122}

The parameter set is $(122 ; 56,55 ; 50)$, with $n=61$. Using the subgroup $H=\{1,9,81,95,119\}$ of $Z_{122}^{\star}$, we give one solution:

$$
\begin{aligned}
J & =\{1,3,6,8,10,13,16,21,23,25,52,61\} \\
K & =\{3,4,6,7,13,19,24,25,46,51,52\}
\end{aligned}
$$

\subsection{Periodic Golay pairs of length 164}

The parameter set is $(164 ; 81,73 ; 72)$, with $n=82$. Using the subgroup $H=\{1,37,57,133,141\}$ of $Z_{164}^{\star}$, we give three non-equivalent solutions:

$$
\begin{aligned}
J & =\{4,5,6,10,11,12,16,20,23,25,30,33,46,51,60,65,123\} \\
K & =\{3,4,11,13,16,19,20,23,30,33,41,44,46,53,66,82,123\} \\
J & =\{1,3,4,6,10,12,13,16,22,23,25,33,34,39,44,46,123\} \\
K & =\{2,4,5,8,10,11,12,16,17,33,34,39,41,51,65,82,123\} \\
J & =\{4,5,6,8,11,13,20,22,24,30,33,34,39,43,44,65,123\} \\
K & =\{2,8,12,13,20,23,25,30,39,41,43,46,51,60,65,82,123\}
\end{aligned}
$$

Additional periodic Golay pairs of length 164 can be constructed by "multiplying" Golay pairs of length 2 with the known periodic Golay pairs of length 82.

\subsection{Periodic Golay pair of length 202}

The parameter set is $(202 ; 100,91 ; 90)$, with $n=101$. Using the subgroup $H=\{1,87,95,137,185\}$ of $Z_{202}^{\star}$, we give one solution:

$$
\begin{aligned}
J & =\{2,4,9,11,12,13,18,20,22,24,25,26,38,41,50,51,53,55,67,76\} \\
K & =\{1,3,4,6,8,9,11,12,16,17,20,25,39,41,48,52,67,76,101\}
\end{aligned}
$$

\subsection{Periodic Golay pairs of length 226}

The parameter set is $(226 ; 106,105 ; 98)$, with $n=113$. Using the subgroup $H=\{1,49,109,129,141,143,219\}$ of $Z_{226}^{\star}$, we give two non-equivalent solutions:

$$
\begin{aligned}
J & =\{1,3,4,5,6,9,10,15,16,36,40,41,43,78,99,113\} \\
K & =\{5,8,12,13,15,21,22,24,26,33,34,40,43,78,99\}
\end{aligned}
$$




$$
\begin{aligned}
J & =\{3,7,12,13,16,18,20,21,22,40,41,43,55,78,99,113\} \\
K & =\{1,2,3,5,10,13,24,26,36,39,40,41,43,78,99\}
\end{aligned}
$$

\section{Algorithm description}

The algorithm that we used to find the new periodic Golay pairs is a straightforward adaptation of the algorithm we used in [5] to construct D-optimal matrices. First we select a subgroup $H$ of $\mathbf{Z}_{v}^{\star}$ and enumerate the orbits of its action on $\mathbf{Z}_{v}$. To construct an SDS with parameters $(v ; r, s ; \lambda)$ we first generate two files of subsets $X$ and $Y$ of size $r$ and $s$, respectively, of $\mathbf{Z}_{v}$ such that the corresponding binary sequences $A$ and $B$ pass the PSD test, i.e. satisfy the inequalities

$$
\operatorname{PSD}_{A}(i) \leq 2 v, \quad \operatorname{PSD}_{B}(i) \leq 2 v, \quad i=1, \ldots, v / 2 .
$$

See [5, 6] for the precise definition of the PSD function. The subsets $X$ and $Y$ are constructed as suitable unions of the orbits of the action described above. Subsequently we look for a match in the two files, i.e., for two subsets $X$ and $Y$ of size $r$ and $s$ such that the the pair $(X, Y)$ satisfies the condition stated in Definition 1 .

Here are some more specific computational details pertaining to the solution for $v=202$ shown in 3.5. First we ran a program for 7 days to generate a list of about 30 million subsets $X$ of $\mathbf{Z}_{202}$ of size 100. All of these sets were made up of $20 H$-orbits each of size 5. Another 7-day run of the same program generated about 26 million subsets $Y$ of size 91. Each of these subsets was the union of 18 orbits of size 5 and the singleton orbit $\{101\}$. In both runs we collected only the subsets for which the corresponding binary sequences pass the PSD test, see [5, 6]. For each of the sets, say $X$, we recorded in a separate file the multiplicities of the nonzero differences $a-b(\bmod 202)$ with $a, b \in X$. Since these searches were not exhaustive, we applied the transformations $X \rightarrow h \cdot X, h \in H$, to the output of the first run and for each of the resulting sets we recorded the difference multiplicities. This resulted in a much bigger file containing about 300 million cases. In one of the files we replaced the multiplicities $m$ with $\lambda-m=90-m$, and then ran a program to find matching lines in the two multiplicity files. The search produced only two matches but they gave equivalent SDSs. Thus we obtained only one solution.

\section{Closing comments}

As ordinary Golay pairs are also periodic Golay pairs, the Golay numbers are also periodic Golay numbers. The known Golay numbers are exactly the integers $v$ admitting the factorization $v=2^{\alpha} 10^{\beta} 26^{\gamma}$, where the exponents $\alpha, \beta, \gamma$ are nonnegative integers. However, as mentioned earlier, there exist infinitely many periodic Golay numbers which do not admit such factorization. Since all Golay numbers in the range 1,2, ., 100 are known [2], we deduce that $34,50,58,68,72,74,82$ are the only periodic Golay numbers for which we are presently sure that they are not Golay numbers. 
By using the construction (2) and the "multiplication theorem" mentioned in section 2, we deduce the existence of Hadamard matrices of order $2 g v$, where $g$ is a Golay number and $v$ a periodic Golay number.

If $v>1$ is a periodic Golay number then $v$ is even, it is a sum of two squares and satisfies the Arasu-Xiang condition of Theorem 2, We list all numbers in the range 1,2, ., 300 which satisfy these three necessary conditions and for which the question whether they are periodic Golay numbers remains open:

$$
\text { 90, 106, 130, 146, 170, 178, 180, 194, 212, 218, 234, 250, 274, 290, 292, } 298 .
$$

This list may be useful to readers interested in constructing new periodic Golay pairs or finding new periodic Golay numbers.

The results of the preprint of this note [7] (posted on the arXiv), have been already used in [10] for the construction of orthogonal and nearly orthogonal designs for computer experiments.

\section{Acknowledgements}

The authors wish to acknowledge generous support by NSERC. This work was made possible by the facilities of the Shared Hierarchical Academic Research Computing Network (SHARCNET) and Compute/Calcul Canada.

\section{References}

[1] K. T. Arasu, Q. Xiang, On the existence of periodic complementary binary sequences. Des. Codes Cryptogr. 2 (1992), no. 3, 257-.262.

[2] P. B. Borwein, R. A. Ferguson, A complete description of Golay pairs for lengths up to 100, Math. Comput. 73 (2003), no. 246, 967-985.

[3] D. Ž. Đoković, Note on periodic complementary sets of binary sequences, Des. Codes Cryptogr. 13 (1998), 251-256.

[4] D. Ž. Đoković, Cyclic $(v ; r, s ; \lambda)$ difference families with two base blocks and $v \leq 50$. Ann. Comb. 15 (2011), no. 2, 233-254.

[5] D. Ž. Đoković and I. S. Kotsireas, New results on D-optimal Matrices. J. Combin. Designs, 20 (2012), 278-289.

[6] D. Ž. Đoković, I. S. Kotsireas, Compression of periodic complementary sequences and applications, Des. Codes Cryptogr. first published online: 24 July 2013, DOI: $10.1007 / \mathrm{s} 10623-013-9862-\mathrm{z}$

[7] D. Ž. Đoković, I. S. Kotsireas, Some new periodic Golay pairs, arXiv:1310.577v1 [math.CO] 22 Oct 2013 
[8] D. Ž. Đoković, I. S. Kotsireas, D. Recoskie and J. Sawada, Charm bracelets and their application to the construction of periodic Golay pairs. arXiv:1405.7328 [math.CO]

[9] D. Ž. Đoković and I. S. Kotsireas, Periodic Golay pairs of length 72 (in preparation).

[10] S. D. Georgiou, S. Stylianou, K. Drosou and C. Koukouvinos, Construction of orthogonal and nearly orthogonal designs for computer experiments, Biometrika (2014), pp. 17.

[11] C. Koukouvinos and J. Seberry, New weighing matrices and orthogonal designs constructed using two sequences with zero autocorrelation function - a review, Journal of Statistical Planning and Inference 81 (1999) 153-182.

[12] S. Eliahou, M. Kervaire, B. Saffari, A new restriction on the lengths of Golay complementary sequences. J. Combin. Theory A 55 (1990), 49-59.

[13] I. S. Kotsireas, C. Koukouvinos, Periodic complementary binary sequences of length 50. Int. J. Appl. Math. 21 (2008), 509-514.

[14] J. Seberry, M. Yamada, Hadamard matrices, sequences, and block designs. In Contemporary design theory, 431.-560, Wiley-Intersci. Ser. Discrete Math. Optim., Wiley, New York, 1992,

[15] A. Vollrath, A modification of periodic Golay sequence pairs. Indiana University REU, Summer 2008. Available on-line: http://mypage.iu.edu/ worrick/reubook08-1.pdf 